Documentation et bibliothèques

DOCUMENTATION BIBLIOTHEQUES

\title{
Le choix des documents dans les bibliothèques universitaires ou de recherche
}

\section{Gilles Paradis}

Volume 23, numéro 2, juin 1977

URI : https://id.erudit.org/iderudit/1055249ar

DOI : https://doi.org/10.7202/1055249ar

Aller au sommaire du numéro

Éditeur(s)

Association pour l'avancement des sciences et des techniques de la documentation (ASTED)

ISSN

0315-2340 (imprimé)

2291-8949 (numérique)

Découvrir la revue

Citer cet article

Paradis, G. (1977). Le choix des documents dans les bibliothèques universitaires ou de recherche. Documentation et bibliothèques, 23(2), 87-97. https://doi.org/10.7202/1055249ar
Résumé de l'article

La responsabilité du choix des documents est souvent remise en question de nos jours, tant au niveau de la théorie que des options pratiques. Qui, en effet, devrait assumer cette tâche dans les bibliothèques universitaires ou de recherche ? Pour dégager des éléments de solution, il convient d'examiner les avantages et les inconvénients des principales formules proposées : choix des documents par les professeurs, recours à la commande globale ou à

« l'approval plan », choix des ouvrages confié aux bibliothécaires, participation des étudiants.
Tous droits réservés (c) Association pour l'avancement des sciences et des techniques de la documentation (ASTED), 1977
Ce document est protégé par la loi sur le droit d'auteur. L'utilisation des services d'Érudit (y compris la reproduction) est assujettie à sa politique d'utilisation que vous pouvez consulter en ligne.

https://apropos.erudit.org/fr/usagers/politique-dutilisation/ 


\title{
Le choix des documents dans les bibliothèques universitaires ou de recherche
}

\author{
Gilles Paradis \\ Bibliothèque générale \\ Université Laval \\ Ste-Foy'
}

La responsabilité du choix des documents est souvent remise en question de nos jours, tant au niveau de la théorie que des options pratiques. Qui, en effet, devrait assumer cette tâche dans les bibliothèques universitaires ou de recherche? Pour dégager des éléments de solution, il convient d'examiner les avantages et les inconvénients des principales formules proposées: choix des documents par les professeurs, recours à la commande globale ou à "l'approval plan», choix des ouvrages confié aux bibliothécaires, participation des étudiants.

The assigning of responsibility for the choice of documentation is often questioned today both at the theoretical and at the practical level. Who, in effect, should be responsible for this task in university and research libraries? In order to find solutions, the advantages and disadvantages of different alternatives must be studied: professors doing the selecting, blanket orders, books on approval, selection by the librarians, the participation of students.

La responsabilidad de la elección de documentos muchas veces en estos dias está questionada, tanto del lado de teoría como del lado de opciones prácticas. Quien debería en efecto tomar sobre si esta tarea en las bibliotecas universitarias o de busca? Para sacar elementos de solución, hay que examinar las ventajas y los inconvenientes de las principales fórmulas propuestas: elección de documentos por los profesores, recurso al encargo global o al "approval plan" elección de obras confiadas a los bibliotecarios, participación de los estudiantes.

Une évolution importante a marqué, au cours des dernières décennies, le développement des collections dans les bibliothèques universitaires. Ces collections ont pris une expansion considérable au plan numérique, certes, mais aussi au niveau de la diversité des supports de la documentation. II est maintenant question non seulement de livres et de périodiques, mais aussi de cartes, de disques, de diapositives, de microdocuments et même de banques de données.

II n'est donc pas surprenant que le problème de la responsabilité du choix des collections soit à nouveau posé. Qui, dans une bibliothèque universitaire, devrait choisir la documentation? D'aucuns estiment que cette responsabilité incombe aux pro- fesseurs. D'autres pensent qu'il faudrait plutôt confier cette tâche aux bibliothécaires. II s'en trouve aussi pour promouvoir l'usage des systèmes dits "envois d'office" ( «approval plan» ou commande globale), formules qui laissent au fournisseur une plus grande initiative. Enfin, certains auteurs voudraient bien que les étudiants participent davantage au choix des ressources documentaires.

Il est facile, en se limitant à un groupe d'auteurs, de se laisser convaincre de la supériorité d'une formule en particulier. Nous voulons présenter ici une analyse des avantages et des inconvénients de chaque formule et dégager certains éléments de solution. 
Précisons que les termes document, ouvrage, et publication gardent au cours de cette recherche leur sens général et qu'ils ne sont par conséquent nullement limitatifs.

\section{Les professeurs}

Pendant longtemps - et cette politique se perpétue encore de nos jours dans une mesure qu'il est difficile d'évaluer avec précision - le choix de la documentation fut un domaine réservé aux professeurs. Cette formule comporte maints avantages que G.A. Kosa a résumés ainsi:

"Faculty members know their re-
spective fields and their literatures
better than librarians do, and
therefore can judge the value of a
book better; furthermore, teaching
staff know their own library needs
and those of their students better
than any librarian can."

Qui, à dire vrai, connaît mieux qu'un professeur de psychologie les progrès récents et les dernières publications dans le champ du développement cognitif, par exemple, ou encore dans celui des thérapies behavioristes? Connaissance acquise, entre autres, par la lecture de livres et de périodiques, les contacts avec les collègues et la participation aux congrès. Seul il peut aisément identifier la documentation requise par son enseignement et sa recherche, de même que les besoins réels de ses étudiants. La meilleure façon de connaître un milieu est certainement d'y vivre et, à cet égard, le professeur occupe une position stratégique quand vient le moment de choisir la documentation propre au travail universitaire.

Enfin, s'il est vrai que le choix de la documentation est étroitement lié au processus de l'enseignement, on doit s'attendre à ce que le professeur participe activement au choix des ouvrages.

II faut cependant reconnaître que la formule consistant à laisser aux professeurs la responsabilité du développement des collections comporte aussi des inconvé-

1. Gesa Attila Kosa, "Book selection trends in American libraries", Australian Library Journal, vol. 21, no. 10 (November 1972), 417 nients. Les plus manifestes sont sans doute un certain déséquilibre des ressources documentaires et une absence de coordination du développement de la collection. Ces effets négatifs proviennent dans une large mesure de facteurs que l'on peut aisément identifier.

Tout d'abord, même si les professeurs possèdent la compétence pour choisir les publications reliées à leur discipline, il ne s'ensuit pas qu'ils l'exercent de fait. L'expérience montre que la situation varie d'un professeur à l'autre. Certains professeurs suggèrent de façon régulière l'achat d'ouvrages, d'autres occasionnellement, quelques-uns jamais. II en résulte que la collection sera très développée, passable ou complètement négligée, selon le degré d'intérêt que chacun aura apporté à la fonction du choix des documents.

Même en nous en tenant au cas des professeurs qui prêtent leur concours au développement de la collection, quelques distinctions s'imposent encore. En effet, bien qu'ils connaissent la situation présente de la recherche dans leur domaine, ils ne choisissent pas toujours de façon systématique les publications récentes. C'est à partir de leurs lectures ou de leurs contacts personnels qu'ils proposent l'acquisition d'un livre en particulier. Ils consultent aussi les comptes rendus de périodiques et reçoivent la publicité des éditeurs. Peu de professeurs cependant dépouillent les bibliographies nationales courantes comme Canadiana, British National Bibliography ou Bibliographie de la France. II est normal d'ailleurs qu'ils connaissent ou qu'ils utilisent moins que les bibliothécaires ces répertoires bibliographiques. Dans l'hypothèse où deux ou trois professeurs seulement se donneraient la peine de parcourir ces listes courantes d'ouvrages, il ne serait pas pratique de songer à étendre ce système au sein d'une faculté regroupant 30 ou 40 professeurs.

Il est par ailleurs difficile pour les professeurs d'assurer le développement continu de la collection. Quelques-uns s'absentent à l'occasion d'un congé sabbatique, d'autres quittent l'université, acceptent des responsabilités administratives ou encore prennent leur retraite. II s'ensuit qu'un sec- 
teur de la collection fera l'objet d'une attention soutenue durant un certain temps mais sera abandonné ultérieurement. Plusieurs bibliothécaires se sont plaints de cet état de choses, comme en témoigne en particulier l'enquête Preibish. ${ }^{2}$ En outre, on remarque que quelques facultés dépensent leur budget rapidement à la fin de l'année fiscale de crainte de voir diminuer leurs octrois l'année suivante. En pareil cas, il est malaisé de parler de développement continu de la collection, d'autant plus que la tentation est forte d'utiliser à la dernière minute un catalogue de réimpressions, dont les prix sont élevés pour ne pas dire exorbitants.

Si le choix des ouvrages par les professeurs ne s'effectue pas toujours de façon systématique et continue, il traduit souvent en revanche les besoins individuels et cela pour le meilleur et pour le pire. «Reliance on faculty selection, écrit Periam Danton, tends to result ... in the purchase of books on a personal-interest basis. ${ }^{3}$ On trouve la même observation chez d'autres auteurs, notamment chez Hamlin. ${ }^{4}$ L'aspect positif de cette situation est que les ouvrages achetés seront vraisemblablement utilisés. Par contre, un professeur dont la spécialité est forcément restreinte développera abondamment un secteur limité de la collection alors que d'autres domaines seront négligés, problème que nous avons signalé précédemment. Mais surtout, un choix basé sur les seuls besoins individuels ne s'accompagne guère de coordination et de planification, approches dont la nécessité se fait de plus en plus pressante dans le domaine du développement des collections.

Depuis longtemps déjà les bibliothécaires soulignent le danger d'une trop grande dépendance de la bibliothèque par rapport aux facultés, en ce qui a trait au choix des

2. André Preibish, Collections and Collection Development in Canadian University Libraries, Ottawa National Library, Resources Rationalization Division, 1972, p. 23-24.

3. J. Periam Danton, Book Selection and Collections: a Comparison of German and American University Libraries, New York, Columbia University, 1963 , p. 69

4. Arthur T Hamlin, "The book collections of British university libraries: an American reaction», International Library Review, vol. 2, no. 2 (April 1970), 147 documents. Dès 1940, Keyes Metcalf, alors directeur de la Harvard University Library, écrivait: "I have already stated my belief that too much reliance on faculty initiative has been unfortunate, I might also say disastrous. ${ }^{5}$

Les professeurs ont joué, historiquement, un rôle décisif dans le choix des ressources documentaires, rôle qui, à la faveur de diverses circonstances, s'est progressivement amoindri. On a craint, dès lors, de perdre les avantages que présentait cette formule. Aussi quelques bibliothécaires ont-ils jugé à propos d'apporter des précisions. Periam Danton, I'un des principaux promoteurs de l'autonomie des bibliothèques, écrivait dans son ouvrage Book Selection and Collections:

"The evidence and position of this study are not in opposition to faculty participation - to the fullest in faculty book selection in all its aspects. Quite the contrary. The evidence is, rather, against the faculty's ultimate authority and responsability; against the largely unrestricted allocation of funds to the faculty; against too great reliance on the faculty by the library." 6

Plus récemment, Jasper $G$. Schad et Ruth L. Adams rappelaient la nécessité de la participation des professeurs au choix des documents. ${ }^{7}$ Partant du fait qu'une bibliothèque ne peut habituellement pas retenir les services d'un nombre suffisant de spécialistes d'une discipline et que, par ailleurs, les bibliographes ne possèdent pas toujours une connaissance spécialisée des disciplines dont ils sont responsables, Schad et Adams proposent de réintégrer les professeurs dans le processus du choix des ouvrages, mais sous une forme différente. Plutôt que de choisir les publications

5. Keyes Metcalf, The Essentials of an Acquisitions Program, cité par David O. Lane, in "The selection of academic library materials, a literature survey". College and Research Libraries, vol. 29, no. 5 (September 1968), 368

6. J. Periam Danton, Book Selection..., 82

7 Jasper G. Schad and Ruth L. Adams, «Book selection in academic libraries: a new approach". College and Research Libraries, vol. 30 , no. 5 (September 1969), 437-442. 
en tenant compte uniquement de leurs besoins particuliers, les professeurs seraient invités à travailler avec les bibliothécaires à certains projets spécifiques. Ces programmes conjoints, si l'on peut dire, viseraient à renforcer un secteur de la collection, à développer un nouveau domaine et ainsi de suite. Selon Schad et Adams, la faculté pourrait libérer les professeurs de quelques responsabilités ou encore la bibliothèque pourrait payer une partie du salaire des professeurs. Au terme de leur enquête, le professeur et le bibliothécaire rédigeraient un rapport contenant, entre autres, la description de l'état présent de la collection et les recommandations pour les achats éventuels de documents.

La formule proposée par Schad et Adams s'applique facilement quand il s'agit de couvrir la bibliographie rétrospective. On peut se demander cependant si elle serait aussi efficace pour le dépouillement de la bibliographie courante.

Les remarques précédentes nous amènent à penser qu'il serait néfaste d'écarter les professeurs du domaine du choix des ressources documentaires; mais que la responsabilité exclusive, voire même première du choix des ouvrages, leur appartienne, c'est là une autre affaire.

\section{La commande globale et "l'approval plan»}

Depuis plusieurs années maintenant, le recours à la commande globale (blanket order) ou à "l'approval plan» est devenu pratique courante dans le monde des bibliothèques universitaires ou de recherche. Une enquête faite auprès de 79 bibliothèques de l'Association of Research Libraries révélait que 44 des 52 bibliothèques ayant répondu au questionnaire utilisaient, à des degrés divers, la commande globale ou "l'approval plan». ${ }^{8}$

Quelques définitions de l'expression "commande globale" ont été proposées. Nous pouvons retenir celle-ci:

"Broadly speaking a blanket order is a contract between a library and

8. Norman Dudley, "The blanket order", Library Trends, vol. 18, no. 3 (January 1970), 318-319. a dealer or publisher specifying that the dealer or publisher select and supply publications within the scope of their services in accordance with the conditions of the contract. Contracts with dealers outline the needs and interest of the library in considerable detail, noting exclusions and special emphases by subject, bibliographical form, level of difficulty and other criteria as may be appropriate." 9

"L'approval plan», comme son nom le suggère, ajoute le privilège pour la bibliothèque de retourner les ouvrages qui n'entrent pas dans les limites du programme établi.

Si nous nous occupons ici de ce procédé de choix, c'est parce qu'il a des répercussions non seulement sur la quantité mais aussi sur la qualité des ouvrages choisis, accordant au fournisseur une part plus active dans le développement des collections.

Depuis plusieurs années, un débat oppose les tenants de cette formule à ceux qui en soulignent surtout les inconvénients. Aussi apparaît-il opportun d'examiner avec soin les arguments des deux groupes. Considérons d'abord l'usage de la commande globale ou de "l'approval plan" comme méthode d'acquisition; nous l'envisagerons ensuite comme moyen de choix.

\section{L'usage de la commande globale et de "l'approval plan» comme méthode d'acquisition}

Plusieurs bibliothécaires ont souligné que l'usage de la commande globale ou de "l'approval plan» constitue une méthode économique d'acquérir les publications. Comment en serait-il autrement, soutiennent-ils, puisqu'on évite de commander les volumes un par un? Dès lors, la recherche préliminaire, la copie de nombreux bons de commande, la vérification des livres lors de la réception, l'intercalation de fiches sont autant de tâches qui sont réduites sinon

9. Robert Wedgeworth, "Foreign blanket orders: precedent and practice", Library Resources and Technical Services, vol. 14, no. 2 (Spring 1970), 258. 
éliminées dans le cadre du processus d'acquisition. $H$. William Axford a poussé plus loin l'analyse en étudiant le coût des opérations qu'implique l'acquisition d'un ouvrage par la méthode de "l'approval plan». Chiffres en mains, il en arrive à la conclusion que "a well-managed approval plan can save at the minimum one full-time position, with significantly higher savings possible depending upon variances in internal procedures» ${ }^{10}$.

D'aucuns ont répliqué, et notamment Ian W. Thom, que si l'usage de "l'approval plan" éliminait quelques tâches, en revanche il en créait d'autres." Par exemple, il faut assurer un contrôle des volumes entre le moment de leur réception et le moment où ils seront disponibles sur les rayons. Les partisans de "l'approval plan" ont riposté en disant que certains fournisseurs envoient des fiches $3^{\prime \prime} \times 5^{\prime \prime}$ qui contiennent tous les renseignements bibliographiques nécessaires à un tel contrôle.

Au plan de l'économie, le recours à «l'approval plan» présente un avantage additionnel: la bibliothèque peut obtenir du fournisseur un meilleur rabais, étant donné qu'elle se procure une importante quantité de volumes. Dans l'enquête mentionnée précédemment, Axford rapporte une réduction de $7.16 \% .{ }^{12}$ Margaret Dobbyn, bibliothécaire à la Kansas State University, rapporte un escompte moins élevé, soit $3.07 \%$ pour les ouvrages scientifiques et $5.62 \%$ pour les ouvrages en sciences sociales. ${ }^{13}$

En plus d'épargner de l'argent, l'emploi de la commande globale ou de "l'approval plan» permet de gagner du temps, si bien que les nouveaux ouvrages sont disponibles plus rapidement. L'enquête menée par Axford $\mathrm{a}$ indiqué que les publications des

10. H. William Axford, "The economics of domestic approval plan". College and Research Libraries, vol. 32, no. 5 (September 1971), 371.

11. Ian W. Thom, «Some administrative aspects of blanket ordering", Library Resources and Technical Services, vol. 13, no. 3 (Summer 1969), 338342.

12. H. William Axford, "The economics...", 372

13. Margaret Dobbyn, "Approval plan purchasing in perspective". College and Research Libraries, vol. 33, no. 6 (November 1972), 483-484 presses universitaires reçues automatiquement arrivent plus vite à la bibliothèque que si on les commande une par une. ${ }^{14}$ L'étude entreprise par Norman Dudley corrobore ces données. ${ }^{15}$

Que penser de tout cela? Dans l'ensemble, la possibilité d'économiser et de sauver du temps en utilisant la commande globale apparaît plausible. Cependant, il ne faut pas oublier que si le travail est effectué par le fournisseur (fiches, pré-sélection ou autres tâches), la bibliothèque finit par en payer la note un jour ou l'autre.

L'usage de la commande globale et de "l'approval plan» comme méthode de choix

Nous avons déjà eu l'occasion d'observer que la formule de "l'approval plan» laisse au vendeur ou au fournisseur la responsabilité du choix initial, compte tenu des profils d'intérêt établis par les bibliothécaires et les professeurs. Quelques bibliothécaires, Le Roy $\mathrm{C}$. Merritt entre autres, se sont inquiétés du fait de remettre au vendeur une large part de la responsabilité du choix des documents. ${ }^{16}$ Limiter les bibliothécaires à rejeter plutôt qu'à choisir les ouvrages n'équivaut-il pas, selon ces auteurs, à abdiquer une des responsabilités fondamentales du bibliothécaire? La formule de "l'approval" plan», réplique David $O$. Lane, n'élimine pas le processus de décision et de choix des ouvrages par les bibliothécaires. ${ }^{17}$ Cette formule modifie plutôt la nature du choix. En effet, au lieu d'utiliser les bibliographies signalétiques ou la publicité des éditeurs, le bibliothécaire a l'ouvrage entre les mains et il peut, par conséquent, porter un jugement plus pertinent.

14. H. William Axford, "The economics...", 373-375.

15. Norman Dudley, "The blanket order», 321

16. Le Roy C. Merritt et al, "A symposium on approval order plans and the book selection responsibilities of librarians", Library Resources and Technical Services, vol. 12, no. 2 (Spring 1968), 133-145.

17. David O. Lane, Study of the Decision Making Procedures for the Acquisition of Science Library Materials and the Relation of these Procedures to the Requirements of College and University Library Patrons, Chicago, American Library Association, 1967 , chap. 5, p. 2. 
À notre avis, l'avantage primordial de "l'approval plan» réside justement dans cette possibilité d'effectuer un choix qui repose sur l'examen du document lui-même. Ce facteur serait déterminant si le pré-choix du vendeur ne constituait pas, dans une large mesure, le choix proprement dit.

On reconnaît généralement que si les bibliothèques ont le droit de retourner les volumes après examen, elles ne doivent pas, à toute fin pratique, en retourner plus de $10 \% .{ }^{18}$ Dans le cas contraire, le fournisseur et la bibliothèque s'exposent à des pertes financières et à des ennuis pratiques. W.R. Taggart parle même de chiffre idéal de $5 \% .{ }^{19}$

II s'ensuit que l'examen des ouvrages choisis par le vendeur est financièrement nécessaire. II suffit, pour s'en convaincre, de prendre un cas en particulier. Si une bibliothèque reçoit 20,000 volumes par année dans le cadre de "l'approval plan» et qu'elle accepte $10 \%$ d'ouvrages inutiles, elle dépensera sans raison $\$ 30,000$, si on suppose que le coût du livre et de son traitement s'élève à $\$ 15$ au total par unité. Or si la bibliothèque ne retourne pas plus de $10 \%$ des publications, cela signifie que dans $90 \%$ des cas au moins le pré-choix était judicieux. Cela revient à dire que le pré-choix et le choix s'identifient dans la majorité des cas.

Les opinions divergent quant aux conséquences de l'usage des commandes globales. Plusieurs bibliothécaires semblent satisfaits des résultats obtenus, comme en témoigne l'enquête décrite par Arthur L. De Volder. ${ }^{20}$ D'autres bibliothécaires, en revanche, ont manifesté leur mécontentement à plusieurs points de vue. On a dit, par exemple, que l'usage de "l'approval plan" avait pour conséquence d'introduire dans la bibliothèque plusieurs publications d'une valeur douteuse ou qui ne correspondaient

18. À ce sujet, voir Gesa Attila Kosa, "Book selection..., 421

19. William R. Taggart, "Blanket approval ordering a positive approach», Canadian Library Journal, vol. 27 , no. 4 (July-August 1970), 287

20. Arthur L. De Volder, Approval Plans: A Survey, New Mexico Univ., Albuquerque, Zimmerman Library, 1972, p. 6. pas aux besoins réels des usagers. On s'est plaint aussi de ne pas recevoir des ouvrages que les bibliothécaires estimaient importants. Selon Roscoe Rouse, les bibliothécaires de l'Oklahoma State University retournèrent jusqu'à $50 \%$ des publications reçues. En outre, au cours de la même expérience, le fournisseur avait omis d'envoyer 466 des 2,303 ouvrages qui étaient répertoriés dans le Publishers Weekly et que les bibliothécaires jugeaient opportun d'acquérir. ${ }^{21}$ Plus récemment, Mary Lee De Vilbiss a décrit une étude comparant l'usage de "l'approval plan" avec les recommandations des professeurs et des bibliothécaires. Dans le cas de la biologie par exemple, seulement 60 des 212 ouvrages désirés avaient été sélectionnés par le vendeur. ${ }^{22}$

Enfin, à un niveau plus général, certains ont déploré que l'usage abusif des commandes globales ait eu pour résultat de développer des collections universitaires trop semblables. Ettlinger, entre autres, prétend qu'il s'agit là d'une duplication inutile. ${ }^{23}$

On voit donc que certains ont retenu surtout les avantages de l'expérience de "l'approval plan" alors que d'autres en ont plutôt souligné les inconvénients. La compétence ou l'absence de compétence du fournisseur a dû jouer beaucoup dans ces expériences plus ou moins heureuses. Remarquons que certaines bibliothèques ont eu recours aux commandes globales pour des secteurs très déterminés, alors que d'autres ont adopté ce système pour l'ensemble de leurs acquisitions.

Pour être efficace, la formule de «l'approval plan» suppose que l'on détermine

21. Roscoe Rouse, "Automation stops there: a case for man made book collections", College and Research Libraries, vol. 31, no 3 (May 1970), 147. 154.

22. Mary Lee De Vilbiss, "The approval builf collection in the medium sized academic library". College and Research Libraries, vol. 36, no. 6 (November 1975), 490.

23. John R.T. Ettlinger, Nation-Wide Rationalization of Acquisition Policies in Canadian College and University Libraries: Are Total World Coverage and Non-duplication of Resources Part of an Impossible Dream? Ottawa, Canadian Association of College and University Libraries, 1973, p. 2.21. 
avec soin les profils d'intérêt, que l'on choisisse un fournisseur compétent et que I'on examine régulièrement les ouvrages envoyés. L'utilisation de "l'approval plan» se concilie davantage avec une politique libérale d'acquisition, par conséquent dans les secteurs où la bibliothèque commande en abondance.

Cette politique d'abondance, qui est un des principes des commandes globales, a elle aussi fait l'objet d'une controverse qu'on pourrait résumer ainsi: "comprehensiveness versus selectivity". Dans le contexte d'une expansion rapide des universités et des bibliothèques universitaires, les commandes globales répondaient à un besoin de développement. Mais certains ainsi Margit Kraft ${ }^{24}$ et Margaret Dobbyn ${ }^{25}-$ ont jugé que le service des commandes globales dépassait la mesure du nécessaire.

On s'est demandé notamment si les bibliothèques universitaires ou de recherche avaient réellement besoin de tous les ouvrages acquis par l'entremise de "l'approval plan" ou de la commande globale. Kraft cite quelques enquêtes qui montrent le faible taux d'usage de certaines collections acquises de cette manière. ${ }^{26}$ En optant pour l'abondance, a-t-on encore fait observer, les bibliothèques acquièrent et conservent de nombreux ouvrages plus ou moins valables au lieu de ne retenir que les publications dont la qualité est reconnue. Enfin, en augmentant de façon considérable les ressources de la bibliothèque, ne risque-t-on pas de rendre non viables les collections de recherche?

Avouons qu'il n'est pas toujours aisé de connaître avec précision les besoins réels de milliers d'étudiants et de centaines de professeurs. D'autre part, la frontière entre ce qui a de la valeur et ce qui n'en a pas n'est pas toujours facile à tracer. On devrait s'attendre toutefois à ce que les bibliothécaires, dans une certaine mesure du moins, s'acquittent de cette tâche.

24. Margit Kraft, "An argument for selectivity in the acquisition of materials for research libraries". $\mathrm{Li}$ brary Quarterly, vol. 37, no. 3 (July 1967), 284 295.

25. Margaret Dobbyn, "Approval plan...», 480-484

26. Margit Kraft, "An argument...», 292-293.
Quant à la croissance plus ou moins limitée de la collection, elle rejoint à notre sens le problème de la recherche et de l'enseignement universitaires. Dans quelle mesure développera-t-on l'enseignement et la recherche à l'université, voilà la véritable question.

\section{Les bibliothécaires}

Nous arrivons maintenant au rôle que les bibliothécaires ont à jouer dans le choix des collections. Une enquête menée auprès de 70 bibliothèques académiques a montré que, dans 62 des 67 bibliothèques ayant répondu au questionnaire, les bibliothécaires participaient au choix des ouvrages. ${ }^{27}$ Une autre enquête, faite en 1974, présente des résultats semblables. ${ }^{28}$ Ces données ne contredisent pas l'enquête sur l'usage de la commande globale ou de "l'approval plan" citée précédemment, car les deux systèmes peuvent coexister, voire se compléter.

Cette nouvelle tendance à une plus grande autonomie de la bibliothèque s'est traduite de diverses manières, de telle sorte que nous ne sommes pas en présence d'une situation homogène. Dans certains cas, le bibliothécaire responsable d'une bibliothèque départementale se charge du choix des ouvrages. Ailleurs, les bibliothécaires de référence consacrent une part de leur temps au choix des volumes. On trouve également des bibliothèques qui retiennent les services de bibliographes ou même de spécialistes d'une discipline dont la responsabilité est de développer la collection dans une ou plusieurs disciplines.

Une opinion veut que les bibliothécaires ne prennent pas la responsabilité du choix des documents, faute de compétence ou tout au moins en raison d'une compétence inférieure à celle des professeurs. Personne, il est vrai, n'est capable de choisir des ouvrages dans toutes les disciplines ou dans toutes les langues. À cet égard, le bibliothécaire n'est pas plus incompétent

27. Robert Haro, "Book selection in academic libraries". College and Research Libraries, vol. 28, no. 2 (March 1967), 104-106.

28. Andrea M. Burgard, SPEC Collection Development Survey, Association of Research Libraries, Washington D.C. Office of Univ. Library Management Studies, 1974. 
que le professeur ou le vendeur. Au niveau universitaire, le travail du choix des ouvrages, comme celui de la référence d'ailleurs, est un travail spécialisé. Or nul ne peut devenir un savant universel et prétendre connaître toutes les disciplines ou toutes les langues.

Néanmoins, la plupart des bibliothécaires sont familiers avec le vocabulaire d'une ou de deux disciplines particulières. Sans s'adonner à un choix systématique dans tous les domaines spécialisés, les bibliothécaires peuvent se limiter aux secteurs qu'ils connaissent davantage. On n'exige pas de celui qui choisit un ouvrage sur Talleyrand qu'il ait rédigé une thèse sur le diplomate français. En outre, les bibliothécaires sont familiers avec les sources bibliographiques et c'est là un avantage précieux.

D'aucuns voudraient, par ailleurs, que les professeurs choisissent les ouvrages de recherche et les bibliothécaires les livres et périodiques concernant l'enseignement. Cette opinion conduit malheureusement à un cul-de-sac. Si l'on nie aux bibliothécaires le droit ou la capacité de choisir des ouvrages spécialisés ou de recherche, on devra logiquement les écarter de la référence spécialisée. Mais qui alors se chargera de ce service? Le fournisseur? Le professeur? Si les bibliothécaires sont exclus du champ de la référence spécialisée (ou s'ils ne prennent pas la responsabilité qui leur incombe à cet égard), il y a bien des chances qu'un tel service n'existe pas, ce qui est à peu près impensable, puisque la plupart des universités offrent un programme de deuxième et de troisième cycles.

Dans un autre ordre d'idées, certains se sont objectés à la présence des spécialistes d'une discipline. Ne se confinent-ils pas à un secteur trop restreint, opinent-ils? Pas dans les faits semble-t-il, puisque leur domaine d'activité demeure assez large (la littérature anglaise, la bibliographie allemande, la biologie, les mathématiques, etc.). Au surplus, les spécialistes possèdent normalement une formation générale.

Enfin, il reste la question financière. En confiant aux bibliothécaires, sous une forme ou sous une autre, le choix des documents, la bibliothèque investit un montant important chaque année au chapitre des traitements. Hamlin écrit à ce sujet: "The machinery represented by this corps of scholars would use an immense amount of financial power that might better be applied to actual purchases. $»^{29} \mathrm{Ce}$ à quoi Danton réplique: "Of course, the more money spent for books, the better, so why not eliminate the cataloguing staffs, too, and use the savings for book purchases? "30

Par contre, les tenants de la participation des bibliothécaires au choix des collections avancent des arguments tout aussi valables. Bien souvent, la bibliothèque universitaire possède la responsabilité finale du développement de la collection et le contrôle du budget. Les bibliothécaires devraient donc avoir beaucoup à dire en ce qui a trait au choix des livres ou autres documents. De plus, les bibliothécaires ont le souci de développer la collection de façon continue et équilibrée.

Quelques enquêtes faites à partir des statistiques du prêt ou à l'aide de listes de contrôle semblent montrer que les bibliothécaires ne se débrouillent pas si mal quand ils choississent eux-mêmes les ouvrages. Edward Evans, notamment, a cherché à établir une relation entre l'usage des documents et le groupe responsable de leur choix.

"The primary finding of this study is that, for the institutions studied, there is a real (statistically significant) difference in pattern of use of current-imprint English-language monographs. The differences exist in the order predicted; that is $\mathrm{li}$ brarians selected more titles that were used than did faculty members or book jobbers, and faculty members selected more titles that were used than did book jobbers." 31

29. Arthur T Hamlin, "The book collections...", 150

30. J. Periam Danton, "University library book selection policy revisited", International Library Review, vol. 3, no. 1 (January 1971), 64.

31. G. Edward Evans, "Book selection and book collection usage in academic libraries", Library Quarterly, vol. 40, no. 3 (July 1970), 301 
Au total 6,891 monographies de langue anglaise ont été examinées dans quatre bibliothèques. Le pourcentage d'ouvrages empruntés et acquis par l'intermédiaire de "l'approval plan», des professeurs et des bibliothécaires s'établissait respectivement à $62 \%, 70 \%$ et $80 \%$.

D'autres enquêtes, par l'utilisation de listes de contrôle, ont démontré le choix généralement judicieux des bibliothécaires.

"The frequently cited results of the Waples-Lasswell study are worth repeating in this connection. Of nearly five hundred English, French, and German works in the fields of the social sciences judged by specialists in those fields to be of primary scholarly importance, Harvard held 63 percent, and the universities of Chicago, California, and Michigan, 49, 40, and 31 percent respectively. The New York Public Library, on the other hand, where book selection is, of course, entirely the responsibility of a corps of subject specialist librarians, held 92 percent. For five hundred seventy-three book and journal titles combined, the New York Public Library, Harvard, Chicago, California, and Michigan held 92,68, 57, 45, and 36 percent respectively." 32

Cette étude, entreprise en 1936, est devenue, dans une certaine mesure, périmée. Elle n'en demeure pas moins significative au sens où elle fut menée au moment où le choix par les professeurs était particulièrement populaire.

Plus récemment, une enquête faite auprès d'une vingtaine de bibliothèques scientifiques, particulièrement dans les domaines de la chimie, de la biologie et de la physique, aboutissait entre autres à la conclusion suivante:

"Comparison of library dominant and faculty dominant institutions on objective collection measures indicates generally better perform- ance by the library dominant institutions. "'33

Toutes ces enquêtes comportent, bien sûr, des limites et il faut éviter une généralisation hâtive. Néanmoins ces données constituent des indices que nous aurions tort de négliger.

\section{Les étudiants}

Tentant de dégager les avantages et les inconvénients de la participation des professeurs, des fournisseurs et des bibliothécaires au choix des documents, nous avons négligé jusqu'à maintenant un autre aspect de la question, soit la contribution des étudiants. II est vrai qu'on mentionne rarement les étudiants dans le débat sur le choix des ouvrages, et l'on peut s'en étonner.

Nancy Bukeye cependant a jugé bon de soulever cette question, mais en se limitant aux étudiants du permier cycle. ${ }^{34}$

Selon elle, la bibliothèque du premier cycle doit satisfaire les besoins des étudiants plutôt que la recherche des professeurs. En outre, les étudiants forment la majorité des usagers de la bibliothèque et en s'acquittant des frais de scolarité, ils fournissent à la bibliothèque les crédits nécessaires à son fonctionnement. Enfin, la participation des étudiants au choix des documents susciterait chez eux une meilleure connaissance et une utilisation plus fréquente de la collection. Pour toutes ces raisons, Nancy Bukeye estime que les étudiants devraient disposer, pour le choix des livres, d'un budget égal à celui des professeurs. Trois bibliothécaires, W. Carl Jackson, Richard D. Johnson et Anne C. Edmonds ont commenté cette opinion, et l'on pourra consulter le même numéro de $\mathrm{Li}$ brary Resources and Technical Services pour examiner le détail de la discussion.

33. Alfred J. Farina and Monroe B. Snyder, DecisionMaking in the Selection of Science Library Materials for Higher Education: Empirical Findings and Guidelines, Chicago, III. American Library Association, 1967, p. 132.

34. Nancy Buckeye, "A plan for undergraduate participation in book selection", Library Resources and Technical Services, vol. 19, no. 2 (Spring 1975), 121-125. 
Nous allons élargir la question et l'envisager de manière plus générale, c'est-àdire pour tous les étudiants et non seulement pour ceux du premier cycle.

II est indéniable que les étudiants forment la majorité des usagers de la bibliothèque. II ne fait pas de doute non plus que ceux des deuxième et troisième cycles utilisent autant, sinon davantage, la collection que les professeurs. Un étudiant qui prépare un mémoire ou une thèse consulte souvent des dizaines de documents.

D'autre part, les étudiants paient leurs frais de scolarité et attendent en retour un service. Or la bibliothèque constitue justement l'un de ces services et son rôle consiste à fournir la documentation nécessaire. On imagine mal qu'elle puisse s'acquitter de cette tâche sans connaître les besoins des étudiants. Cela ne signifie pas que les étudiants doivent prendre la responsabilité du développement de la collection, mais qu'ils doivent participer d'une certaine manière au choix des ouvrages. N'avons-nous pas trop souvent supposé que les professeurs et les bibliothécaires connaissaient ipso facto les besoins des étudiants?

Mais peut-être le problème ne se situet-il pas tant au niveau du principe de la participation des étudiants qu'au niveau des mécanismes mis en place pour réaliser cette participation. Bien sûr, il existe déjà des formes de communication entre les étudiants d'une part et les professeurs et les bibliothécaires d'autre part. Mais avouons qu'il y a ici place pour des idées neuves et qu'il faudra bien un jour dépasser le stade de la boîte à suggestions.

\section{Commentaires et conclusion}

En parcourant les diverses méthodes proposées pour le choix des ouvrages, nous avons pu déceler les avantages et les inconvénients de chacune. Selon l'importance que nous accordons à certains éléments plutôt qu'à d'autres, et compte tenu des particularités financières, géographiques et linguistiques, notre jugement peut être différent. Une bibliothèque qui dispose d'un budget considérable sera plus naturellement portée à utiliser les commandes globales ou à retenir les services de bi- bliothécaires responsables du développement des collections. En revanche, une bibliothèque moins riche comptera davantage sur la collaboration des professeurs.

II nous faut donc, à la limite, examiner chaque cas en particulier. Mais ce jugement, pour être judicieux, implique l'étude des avantages et des désavantages de chaque formule, étude dont nous venons d'esquisser les grandes lignes. En outre, pour jauger à leur juste valeur les différents arguments, il convient de les considérer à la lumière de quelques éléments. Ces éléments portent plus sur le cadre dans lequel il faut chercher la solution que sur la solution elle-même.

Premièrement, un des critères essentiels pour juger du choix des ouvrages consiste à savoir s'il a été bien ou mal fait.

Deuxièmement, et c'est un point sur lequel nous voulons attirer l'attention, nous avons tout à gagner à relier le choix des ouvrages au but et aux objectifs que poursuit la bibliothèque universitaire. Or il est de plus en plus évident que la bibliothèque ne peut plus se limiter à acquérir et à organiser les ressources documentaires. Elle doit devenir un véritable service à la clientèle. C'est en fonction de cet objectif majeur que nous devons considérer aujourd'hui la question du choix des collections.

Troisièmement, les quatre méthodes décrites plus haut ne sont pas exclusives; le choix par les bibliothécaires et l'usage de la commande globale, par exemple, peuvent coexister.

Quatrièmement, si les méthodes de choix des publications ne sont pas exclusives, il n'en demeure pas moins qu'une méthode aura normalement préséance sur les autres. Au fond, il s'agit de savoir qui sera d'abord responsable du choix des ouvrages. Compte tenu de l'analyse précédente, nous croyons que la responsabilité première du choix des documents devrait revenir aux bibliothécaires.

On conviendra qu'il existe présentement un besoin manifeste de coordination et de planification dans le domaine du dé- 
veloppement des collections. II faut, par exemple, établir un équilibre entre la collection de recherche et celle de l'enseignement. Cet équilibre doit également se retrouver au niveau des besoins souvent très différents des facultés. II est nécessaire aussi que quelqu'un s'occupe du développement continu et systématique de la collection en plus d'assurer la collaboration avec les autres bibliothèques universitaires. Or le bibliothécaire est le seul pour qui le choix des documents n'est pas une activité secondaire ou même marginale. Tout cela ne signifie pas que les bibliothécaires devraient agir seuls, mais plutôt qu'ils devraient être les premiers responsables.

Par ailleurs, le recours à la commande globale et à "l'approval plan» s'applique surtout, ainsi que nous l'avons constaté, dans les domaines où la bibliothèque commande en abondance ou encore dans le cas des pays dépourvus d'une organisation bibliographique suffisante. Le fournisseur doit s'occuper de la rentabilité de son entreprise et le profit demeure pour lui un objectif majeur, parfaitement compréhensible d'ailleurs. Cela ne l'empêche pas d'offrir un service et, dans certains cas, un service très efficace. Le fournisseur reste quand même loin des professeurs et des étudiants qui finalement utilisent la collection. Toutefois, si les bibliothécaires rédigent avec soin le profil d'intérêt et s'ils examinent régulièrement les ouvrages envoyés, "l'approval plan» peut s'avérer utile.

Considéré à la lumière du but que poursuit la bibliothèque, le problème de la responsabilité du choix des documents s'éclaire singulièrement. S'il est vrai que la bibliothèque doit être avant tout un service, on s'attendra tout naturellement à trouver entre les bibliothécaires et les usagers une communication régulière et efficace. Si notre analyse est exacte, les professeurs et les étudiants participeront d'autant plus au choix des publications qu'il y aura un bibliothécaire en contact avec leur faculté. Prenons par exemple le cas des professeurs. Certains d'entre eux s'occupent du choix des publications quel que soit le système de choix adopté. À l'opposé, nous retrouvons les professeurs qui ne se mêleront jamais de choisir les ouvrages même si vous les enjoignez de le faire par voie de subpoena. Enfin, on rencontre un troisième groupe de professeurs, probablement les plus nombreux, qui participeront au choix des ouvrages pourvu que la bibliothèque crée les conditions favorables. Or le bibliothécaire est justement en mesure de jouer ce rôle de catalyseur. En contact avec les professeurs et les étudiants, il peut obtenir leur collaboration et cela au bénéfice de tous les intéressés. Au surplus, les professeurs et les étudiants savent à qui s'adresser s'ils veulent suggérer l'achat d'un ouvrage.

On comprendra alors pourquoi il existe entre le service de référence et celui du développement des collections un lien très étroit. Quand un bibliothécaire choisit des ouvrages en fonction des gens qui le consultent au niveau de la recherche documentaire, la collection répondra probablement aux besoins réels des usagers. L'idéal est donc de confier aux mêmes bibliothécaires le choix des documents et la référence.

Dans cette perspective, le choix des ouvrages a des chances d'être bien fait. Informé des besoins réels de la clientèle et connaissant les publications nouvelles, le bibliothécaire est en mesure d'effectuer un choix judicieux. S'il omet d'acquérir une publication utile, les usagers le lui signaleront sans doute au niveau de la référence. D'autre part, le bibliothécaire informera l'usager des nouvelles acquisitions susceptibles de l'intéresser.

Envisagée de ce point de vue, la question de la responsabilité du choix des collections nous apparaît sous un angle un peu différent. Les diverses méthodes de choix ont bel et bien des avantages et des inconvénients. Mais en reliant la question du choix des documents aux objectifs de la bibliothèque, il devient non plus seulement possible mais encore souhaitable de confier à une équipe de bibliothécaires la responsabilité première du choix des ouvrages. 